\title{
Primera cita de Lestes dryas Kirby, 1890 (Odonata, Lestidae) para Gipuzkoa (País Vasco, España).
}

\section{First record of Lestes dryas Kirby, 1890 (Odonata, Lestidae) from Gipuzkoa (Basque Country, Spain).}

Iñaki Mezquita-Aranburu' ${ }^{1}$

*

\section{Resumen}

Se recoge la primera cita para Gipuzkoa (País Vasco, España) de Lestes dryas Kirbyi, 1890 (Odonata, Lestidae).

Palabras clave: Odonata, Lestidae, Lestes dryas, Alabita, Eskoriatza, Gipuzkoa, Euskadi, España.

\section{Abstract}

The first record of Lestes dryas Kirbyi, 1890 (Odonata, Lestidae) in Gipuzkoa (Basque Country, Spain) is reported.

Key words: Odonata, Lestidae, Lestes dryas, Alabita, Eskoriatza, Gipuzkoa, Basque Country, Spain.

\section{Laburpena}

Lestes dryas Kirbyi, 1890 (Odonata, Lestidae) espeziearen lehen aipua jaso da Gipuzkoarako (Euskadi, Espainia).

Gako hitzak: Odonata, Lestidae, Lestes dryas, Alabita, Eskoriatza, Gipuzkoa, Euskadi, Espainia.

\footnotetext{
1 Departamento de Entomología Sociedad de Ciencias Aranzadi Zientzia Elkartea Zorroagagaina 11, 20004 Donostia-San Sebastián (Spain) 
Lestes dryas Kirby, 1890 es un léstido de mediano tamaño que suele habitar en gran variedad de aguas estancadas con cierta acidez y de escasa profundidad que típicamente se secan en verano o que tienen orillas poco profundas, ofreciendo micro hábitats cálidos a las larvas que las habitan (Askew, 2004; Dijkstra \& Lewington, 2006). Está ampliamente distribuida por Europa, aunque ausente en el norte de Fenoscandia y más diseminada en la región Mediterránea, estando ausente en la mayoría de las islas del Mediterráneo. En general es común en las partes bajas de centro Europa, pero en el sur de su área de distribución las mayores poblaciones se dan a mayores altitudes (Boudot \& Kalkman, 2015). La especie ocupa un amplio rango de altitud, aunque parece tener una distribución preferentemente montana o subalpina en Asturias (Ocharan \& Torralba-Burrial, 2004), que coincidiría con la tipología de la localidad en la que se ha encontrado en Gipuzkoa.

Hasta la fecha no se había encontrado esta especie en las provincias vascas litorales (Mezquita \& Ocharan, 2012; Prunier et al., 2015). En Álava está ben distribuida, siendo más frecuente en sus zonas media y sur (Gainzarain, 2018).

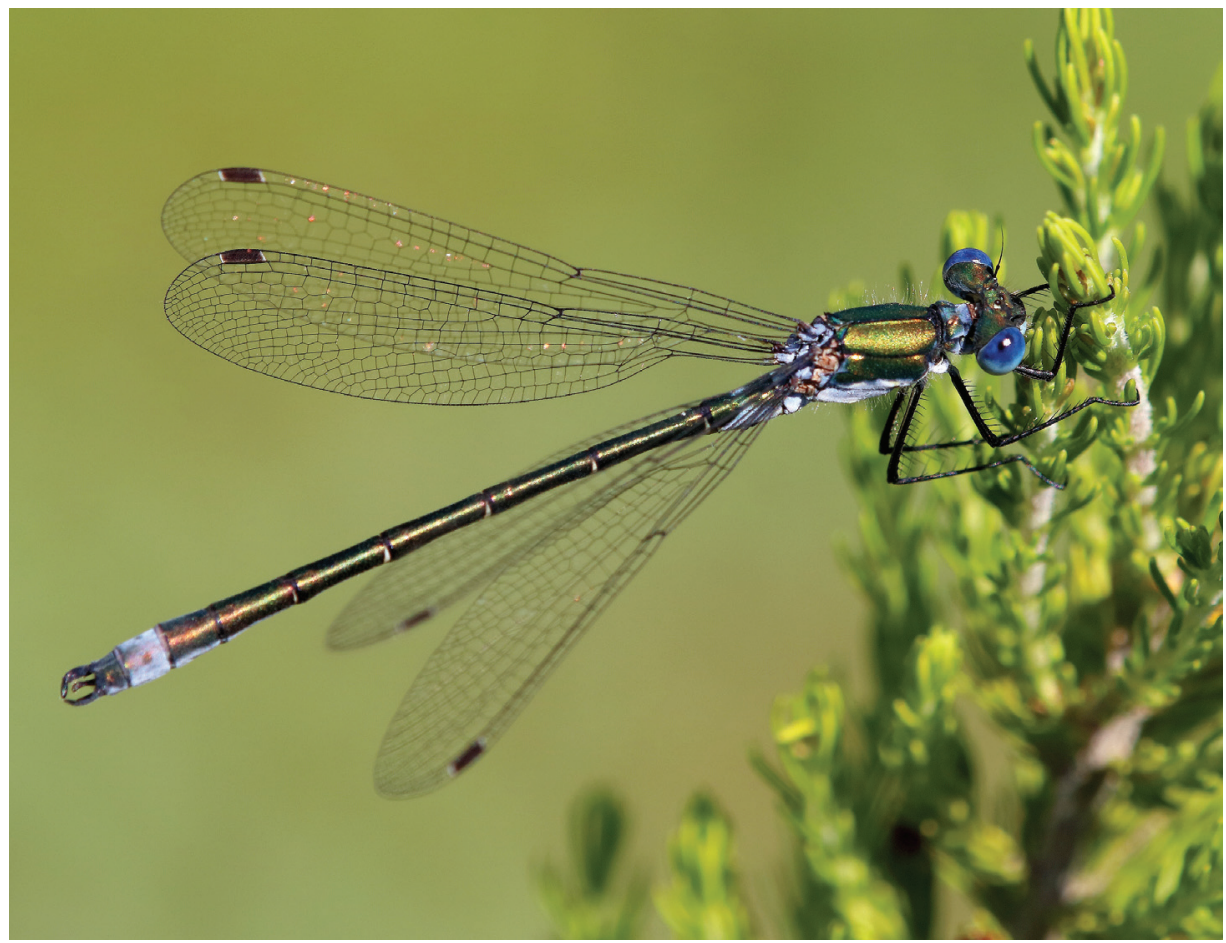

Fig. 1.- Macho de Lestes dryas localizado en Alabita (Eskoriatza, Gipuzkoa) (foto de IMA).

Fig. 1.- Male of Lestes dryas observed in Alabita (Eskoriatza, Gipuzkoa) (photo by IMA). 
El 11 de junio de 2017, durante una salida conjunta de miembros del Departamento de Herpetología con el autor, se visitaron a la una del mediodía (hora local) dos charcas situadas en el paraje conocido como Alabita (UTM 30TWN4057, altitud 940 m.), perteneciente al municipio de Eskoriatza (Gipuzkoa), dentro del Parque Natural Aitzkorri-Aratz. En la primera de las charcas se localizaron varios machos de la especie compartiendo espacio con varios ejemplares de Pyrrhosoma nymphula y un macho de Anax imperator, a las que hay que añadir Coenagrion puella en la segunda charca. En esta segunda charca, se localizó una pareja de la especie en cópula y, posteriormente, en puesta; siendo esta hembra la única observada en esta visita. Posteriormente, se ha constatado la presencia de varias hembras por parte de Iker Novoa Fariñas (com. pers.). En las proximidades de las charcas, en varios arroyos, se encontraron además ejemplares de las especies: Calopteryx virgo, Coenagrion puella, Coenagrion scitulum, Pyrrhosoma nymphula, Lestes sponsa, Anax imperator, Libellula depressa y Orthetrum coerulescens.

Con esta cita, el catálogo de odonatos encontrados en Gipuzkoa se eleva a 47 especies (Mezquita-Aranburu \& Ocharan, 2012; Paz \& Conesa, 2017; Miralles et al. 2017).

\section{Agradecimientos}

A Martiño Cabana por sus valiosas aportaciones en la redacción de esta nota. A Iker Novoa Fariñas por su inestimable ayuda, así como a los miembros del Departamento de Herpetología de la Sociedad de Ciencias Aranzadi Zientzi Elkartea por su ayuda y grata compañía.

\section{Bibliografía}

Askew, R.R. 2004. The dragonflies of Europe (revised edition). Harley Books. Colchester.

Boudot, J.P., Kalkman, V.J. 2015. Atlas of the European dragonflies and damselflies. KNNV Publishing. The Nederlands.

Dijkstraa, K-D.B., Lewington, R. 2006. Field guide to the dragonflies of Britain and Europe. British Wildlife Publishing. Dorset.

Gainzarain, J.A., Ocharan, F.J. \& Mezquita, I. 2013. Catálogo de los odonatos (Insecta: Odonata) de Álava, norte de España. Bol. Soc. Entomol. Aragonesa 53: 173-185.

Mezquita-Aranburu, I., Ocharan, F.J. 2012. Odonatos de Gipuzkoa, Munibe, Cienc. nat. 60: 51-75. 
Miralles-Núñez, A., Obregón-Romero, R., Mezquita-Aranburu, I. 2017. Nuevos registros sobre la distribución y reproducción de Trithemis kirbyi Sélys, 1891 (Odonata: Libellulidae) en la Península Ibérica. Bol. Soc. Entomol. Aragonesa 61: 241-244.

Ocharan, F.J., Torralba-Burrial, A. 2004. La relación entre los odonatos y la altitud: el caso de Asturias (Norte de España) y la Península Ibérica (odonata). Bol. Soc. Entomol. Aragonesa 35: 103-116.

Paz-Leiza, L., Conesa, M. 2017. Primera cita y confirmación de reproducción de Gomphus vulgatissimus (Linnaeus, 1758) (Odonata: Gomphidae) en Gipuzkoa (País Vasco, España). Munibe, Cienc. nat. 65: 141-144.

Prunier, F., Brotóns, M., Cabana, M., Campos, F., Casanueva, P., Chelmick, D., Cordero Rivera, A., Díaz Martínez, C., Evangelio, J.M., Gainzarain, J. A., García-Moreno, J., Lockwood, M., de los Reyes, L., Mañani, J., Mezquita-Aranburu, I., Muddeman, J., Ocharan, F.J., Otero Pérez, F., Prieto-Lillo, E., Requena, C., Ripoll, J., Rodríguez Luque, F., Rodríguez, P., Romeo, A., Salcedo, J., Salvador Vilariño, V., Sánchez Balibrea, J., Tamajón Gómez, R., Torralba-Burrial, A., Tovar, C., Winter, P., Zaldívar, R. 2015. Actualización del inventario provincial de Odonatos de España peninsular e Islas Baleares. Bol. ROLA 6: 59-84.

\section{है}

Fecha de recepcion/Date of reception: 28/01/2019

Fecha de aceptacion/Date of acceptance: 17/02/2019

Editor Asociado/Associate editor: Alberto Castro 